\title{
A geometrical approach to nonlinear perturbations in relativistic cosmology
}

\author{
David Langlois ${ }^{a}$, Filippo Vernizzi ${ }^{b}$ \\ a APC (CNRS-Université Paris 7), 10 rue Alice Domon et Léonie Duquet, 75205 Paris Cedex 13, France and \\ ${ }^{b}$ CEA, IPhT, 91191 Gif-sur-Yvette cédex, France, \\ CNRS, URA-2306, 91191 Gif-sur-Yvette cédex, France
}

\begin{abstract}
We give a pedagogical review of a covariant and fully non-perturbative approach to study nonlinear perturbations in cosmology. In the first part, devoted to cosmological fluids, we define a nonlinear extension of the uniform-density curvature perturbation and derive its evolution equation. In the second part, we focus our attention on multiple scalar fields and present a nonlinear description in terms of adiabatic and entropy perturbations. In both cases, we show how the formalism presented here enables one to easily obtain equations up to second, third and higher orders.
\end{abstract}

\section{INTRODUCTION}

The relativistic theory of cosmological perturbations (see e.g. [1-7]) is an indispensable tool to interpret cosmological data such as the Cosmic Microwave Background (CMB) anisotropies, and thus to connect the scenarios of the early universe, such as inflation, to cosmological observations. Because the temperature anisotropies of the CMB are so small $\left(\delta T / T \sim 10^{-5}\right)$, considering only linear perturbations is an excellent first approximation and this is why most of the efforts devoted to the study of cosmological perturbations have dealt with the linear theory.

However, with the rapidly increasing precision of cosmological data, we have now reached a stage where nonlinear features of primordial perturbations could be observationally accessible. In the last few years, this has motivated an intensive development of the theory of cosmological perturbations beyond linear order. Two main strategies have been considered. The first one is based on extending the traditional coordinate-based approach by adding secondorder perturbations in the metric and in the matter fields and by computing the equations of motion for the metric and matter perturbations by brute force $[6,8-12]$. The second strategy is based on a fully nonlinear treatment, but restricted to super-Hubble scales and working directly in a coordinate system [13-19]. This strategy is related to the so called separate universe picture that represents our universe, on scales larger than the Hubble radius, as juxtaposed Friedmann-Lemaitre-Robertson-Walker (FLRW) universes with slightly different scale factors [20, 21].

The approach that we present here, based on [22-25], relies on a geometrical perspective. From a computational point of view, it can be seen as a "middle way" between the two main strategies discussed above. Inspired by the so-called covariant formalism [26, 27] for cosmological perturbations developed in [28-33] (see also the article by R. Maartens in this issue [34]), the fundamental idea is to use a nonlinear and covariant approach as long as possible and try to construct covariant quantities that mimic the traditional quantities that have been useful in the theory of linear perturbations (see also [35] for an alternative application of the covariant formalism to nonlinear perturbations). Since these quantities are tensors, they are intuitively much easier to understand from a geometrical point of view. Once these covariant objects have been defined and their evolution equation obtained in a fully nonlinear and covariant form, it is then possible to choose a coordinate system and to expand the quantities and equations to the desired order, in order to make concrete quantitative calculations.

In this review we discuss two examples of this approach which are particularly illuminating. The first is the nonlinear extension of the familiar linear curvature perturbation on uniform-density hypersurfaces, usually denoted $\zeta$, introduced in [36]. This quantity plays a crucial role in the linear theory, because it is conserved on large scales for adiabatic perturbations [37,38], and therefore enables one to very easily relate cosmological perturbations in the very early universe and in the late universe, as long as the scales remain super-Hubble. The second example is useful in the context of multi-field inflation. In the linear theory, it is useful to decompose the perturbations of the scalar fields into (instantaneous) adiabatic and entropic modes, which are simply the projections of the perturbations along directions that are, respectively, tangential and orthogonal to the background trajectory in field space. Once again, it is possible to generalize this decomposition into adiabatic and entropic modes at the nonlinear level. This can be used to compute the evolution of nonlinear perturbations and the resulting non-Gaussianities.

This review is divided into two parts. In the next section we consider the treatment of a cosmological fluid, while the third, and final, section is devoted to the case of several scalar fields. 


\section{COSMOLOGICAL FLUIDS}

\section{A. Covariant approach}

In this section we consider a single perfect fluid, characterized by a comoving four-velocity $u^{a}\left(u_{a} u^{a}=-1\right)$, a proper energy density $\rho$ and a pressure $P$. The energy-momentum tensor associated to the perfect fluid is given by

$$
T_{b}^{a}=(\rho+P) u^{a} u_{b}+P g_{b}^{a}
$$

To fully characterize the fluid, one needs an equation of state relating $P$ to $\rho$ and, possibly, to other physical quantities if the fluid is not barotropic.

The spatial projection tensor orthogonal to the fluid velocity $u^{a}$ is defined by

$$
h_{a b}=g_{a b}+u_{a} u_{b}, \quad\left(h_{b}^{a} h_{c}^{b}=h_{c}^{a}, \quad h_{a}^{b} u_{b}=0\right) .
$$

It is also useful to introduce the familiar decomposition

$$
\nabla_{b} u_{a}=\sigma_{a b}+\omega_{a b}+\frac{1}{3} \Theta h_{a b}-a_{a} u_{b}
$$

where one finds on the right-hand side the (symmetric) shear tensor $\sigma_{a b}$, the (antisymmetric) vorticity tensor $\omega_{a b}$, the volume expansion $\Theta \equiv \nabla_{a} u^{a}$ and the acceleration $a_{a} \equiv u^{c} \nabla_{c} u^{a}$.

The integration of $\Theta$ along the fluid world lines with respect to the associated proper time $\tau$,

$$
\alpha \equiv \frac{1}{3} \int d \tau \Theta
$$

can be used to define, for each observer comoving with the fluid, a local scale factor $S=e^{\alpha}$. It follows that $\Theta=3 \dot{\alpha}$, where the dot of a scalar quantity denotes its derivative along $u^{a}$, i.e. $\dot{\alpha} \equiv u^{a} \nabla_{a} \alpha$. This quantity $\alpha$, which can be seen as a (covariantly defined) local number of e-folds, plays a crucial rôle in the definition of our covariant and nonlinear extension of the familiar linear curvature perturbation on uniform-density hypersurfaces, usually denoted $\zeta$, introduced in [36].

Our starting point is the conservation of the energy-momentum tensor,

$$
\nabla_{a} T_{b}^{a}=0
$$

which yields, after substituting (1) and projecting along $u^{b}$, the continuity equation

$$
\dot{\rho}+3 \dot{\alpha}(\rho+P)=0
$$

The spacetime gradient of this expression can be written as

$$
\left(\partial_{a} \rho\right)^{\cdot}+3\left(\partial_{a} \alpha\right)^{\cdot}(\rho+P)+3 \dot{\alpha} \partial_{a}(\rho+P)=0
$$

where the dot acting on a spacetime gradient, which is a covector, is defined as the Lie derivative (see e.g. [39]) with respect to $u^{a}$. For any covector $X_{a}$, this means

$$
\dot{X}_{a} \equiv \mathcal{L}_{u} X_{a} \equiv u^{b} \partial_{b} X_{a}+X_{b} \partial_{a} u^{b}
$$

More generally, a dot acting on any tensor will denote the Lie derivative of this tensor with respect to $u^{a}$. This definition is compatible with our notation for the scalars since the Lie derivative coincides with the derivative along $u^{a}$ for scalars (and we have used the property $\partial_{a}(\dot{\rho})=\left(\partial_{a} \rho\right)^{\cdot}$ to obtain $(7)$ ).

After some simple manipulations, one finds that Eq. (7) is equivalent to [22, 23]

$$
\dot{\zeta}_{a}=-\frac{\Theta}{3(\rho+p)}\left(\partial_{a} p-\frac{\dot{p}}{\dot{\rho}} \partial_{a} \rho\right),
$$

where the left-hand side consists of the time derivative, i.e. the Lie derivative with respect to $u^{a}$, of the covector

$$
\zeta_{a} \equiv \partial_{a} \alpha-\frac{\dot{\alpha}}{\dot{\rho}} \partial_{a} \rho .
$$


This covector can also be re-expressed, using (6), in the form

$$
\zeta_{a}=\partial_{a} \alpha+\frac{\partial_{a} \rho}{3(\rho+P)}
$$

and, if $w \equiv P / \rho$ is constant, this is a total gradient since

$$
\zeta_{a}=\partial_{a}\left[\alpha+\frac{1}{3(1+w)} \ln \rho\right] \quad(w=P / \rho=\text { const })
$$

On the right-hand side, the quantity

$$
\Gamma_{a} \equiv \partial_{a} P-\frac{\dot{P}}{\dot{\rho}} \partial_{a} \rho
$$

is a nonlinear generalization of the non-adiabatic pressure. It vanishes for purely adiabatic perturbations, for instance when the pressure $P$ is solely a function of the density $\rho$.

Eq. (9) has a form very similar to the conservation equation for $\zeta$ of the linear theory, which will be rederived in the next section. At the non-linear level, our covector $\zeta_{a}$ is closely related to the non-linear perturbation introduced in [19],

$$
\zeta=\delta N+\frac{1}{3} \int_{\bar{\rho}}^{\rho} \frac{d \tilde{\rho}}{\tilde{\rho}+\tilde{P}}
$$

where $\bar{\rho}(t)$ is the homogeneous background density, while $\rho(t, \vec{x})$ is the local energy density and $N=\int d t \Theta / 3$ is the number of e-folds, defined with respect to the time coordinate $t$. A similar non-linear quantity has been introduced earlier in [17]. The advantage of the definition (10) is that one does not need to introduce a coordinate system or to restrict oneself to super-Hubble scales, and that its evolution equation (9) is exact, fully non-perturbative and valid at all scales. Moreover, it is worth stressing that this equation is a direct consequence of the conservation of the energy-momentum tensor and is independent of the underlying theory of gravitation.

\section{B. Link with the coordinate approach}

We now relate the covariant approach with the more familiar coordinate based formalism [1-7]. We first examine the linear perturbations and consider later the perturbations at second and third orders.

\section{Linear theory}

A cosmological spacetime closed to FLRW geometry can be described by the perturbed metric

$$
d s^{2}=-(1+2 A) d t^{2}+2 a B_{i} d x^{i} d t+a^{2}\left(\gamma_{i j}+H_{i j}\right) d x^{i} d x^{j},
$$

where $a=e^{\bar{\alpha}}$ is the background scale factor. As usual, one can decompose the linear perturbations into so-called scalar, vector and tensor modes,

$$
\begin{aligned}
B_{i} & =\vec{\nabla}_{i} B+B_{i}^{V}, \\
H_{i j} & =-2 \psi \gamma_{i j}+2 \vec{\nabla}_{i} \vec{\nabla}_{j} E+2 \vec{\nabla}_{(i} E_{j)}^{V}+2 E_{i j}^{T},
\end{aligned}
$$

where $B_{i}^{V}$ and $E_{i}^{V}$ are transverse, i.e., $\vec{\nabla}_{i} B^{V i}=0=\vec{\nabla}_{i} E^{V i}$, and $E_{i j}^{T}$ is transverse and traceless, i.e., $\vec{\nabla}_{i} E^{T i j}=0$ and $\gamma^{i j} E_{i j}^{T}=0$. Here $\vec{\nabla}_{i}$ denotes the three-dimensional covariant derivative with respect to the homogeneous spatial metric $\gamma_{i j}$ (which is also used to lower or raise the spatial indices).

The corresponding matter content is a perfect fluid with perturbed energy density and pressure, $\rho\left(t, x^{i}\right)=\bar{\rho}(t)+$ $\delta \rho\left(t, x^{i}\right), P\left(t, x^{i}\right)=\bar{P}(t)+\delta P\left(t, x^{i}\right)$, and four-velocity

$$
u^{\mu}=\bar{u}^{\mu}+\delta u^{\mu}, \quad \delta u^{\mu}=\left\{-A, v^{i} / a\right\}, \quad v_{k}=\vec{\nabla}_{k} v+v_{k}^{V},
$$

where $v_{i}^{V}$ is transverse, $\vec{\nabla}_{i} v^{V i}=0$. 
It is easy to check that the covector $\zeta_{a}$ automatically vanishes in the background. At linear order, its spatial components are simply $[22,23]$

$$
\zeta_{i}=\partial_{i} \zeta, \quad \zeta \equiv \delta \alpha-\frac{H}{\bar{\rho}^{\prime}} \delta \rho
$$

where $H$ denotes the Hubble rate, $H=a^{\prime} / a=\bar{\alpha}^{\prime}$ and we recall that a prime denotes the partial derivative with respect to the cosmic time $t$. The quantity $\zeta$ defined above differs from the familiar definition [36]

$$
\zeta_{B} \equiv-\psi-H \frac{\delta \rho}{\bar{\rho}^{\prime}}
$$

because $\delta \alpha$ differs from $-\psi$. They are related by [23]

$$
\delta \alpha=-\psi+\frac{1}{3} \nabla^{2} E+\frac{1}{3} \int d t \frac{\nabla^{2} v}{a},
$$

and they coincide when the gradient terms are negligible.

The components of the non-adiabatic term $\Gamma_{a}=\partial_{a} P-(\dot{P} / \dot{\rho}) \partial_{a} \rho$ can be deduced directly from the components of $\zeta_{a}$ by substituting $P$ to $\alpha$. One finds

$$
\Gamma_{i}=\partial_{i} \Gamma, \quad \Gamma \equiv \delta P-\frac{\bar{P}^{\prime}}{\bar{\rho}^{\prime}} \delta \rho .
$$

Putting all together Eq. (9) gives, at first order,

$$
\zeta^{\prime}=\zeta_{B}^{\prime}+\frac{1}{3} \nabla^{2}\left(E^{\prime}+v / a\right)=-\frac{H}{\bar{\rho}+\bar{P}}\left(\delta P-\frac{\bar{P}^{\prime}}{\bar{\rho}^{\prime}} \delta \rho\right) .
$$

For adiabatic perturbations, the right-hand side vanishes and the quantity $\zeta$ is conserved at all scales because it comes directly from the covector $\zeta_{a}$. By contrast, $\zeta_{B}$ is conserved only when the gradient terms are negligible.

\section{Second-order perturbations and beyond}

The covariant approach turns out very useful to go beyond linear order in perturbation theory. Any function $X$ can be decomposed in the form

$$
X\left(t, x^{i}\right)=\bar{X}(t)+\delta X^{(1)}\left(t, x^{i}\right)+\delta X^{(2)}\left(t, x^{i}\right)+\delta X^{(3)}\left(t, x^{i}\right)+\ldots
$$

where a quantity with a superscript ${ }^{(n)}$ is the $n$-th order contribution to $X$ in the perturbation theory expansion [9] (note that here we do not follow the convention of including a numerical factor $1 / n$ ! in front of the $n$-th contribution).

Expanding $\zeta_{i}=\partial_{i} \alpha-(\dot{\alpha} / \dot{\rho}) \partial_{i} \rho$ at second order one finds that, in contrast with the first order expression, $\zeta^{(2)}$ cannot be written as the gradient of a scalar perturbation. Indeed, after some manipulations one finds, at second order [22, 23],

$$
\zeta_{i}^{(2)}=\partial_{i} \zeta^{(2)}+\frac{\delta \rho}{\bar{\rho}^{\prime}} \partial_{i} \zeta^{(1) \prime}
$$

with

$$
\zeta^{(2)}=\delta \alpha^{(2)}-\frac{H}{\bar{\rho}^{\prime}} \delta \rho^{(2)}-\frac{1}{\bar{\rho}^{\prime}} \delta \alpha^{\prime} \delta \rho+\frac{H}{\bar{\rho}^{\prime 2}} \delta \rho \delta \rho^{\prime}+\frac{1}{2 \bar{\rho}^{\prime}}\left(\frac{H}{\bar{\rho}^{\prime}}\right)^{\prime} \delta \rho^{2} .
$$

On large scales, using

$$
\alpha \approx \ln a-\psi-\psi^{2},
$$

one can show that $\zeta^{(2)}$ is directly related to the conserved second-order quantity defined by Malik and Wands [40]: $\zeta^{(2)} \approx \zeta_{\mathrm{MW}}^{(2)}-\left(\zeta_{\mathrm{MW}}^{(1)}\right)^{2}$ (see also the discussion in [41]). We use the symbol $\approx$ to denote an equality only valid on large scales. 
Now that we have identified a second-order expression for $\zeta$, we can expand Eq. (9) at second order. On making use of the second line of Eq. (8) to reexpress the Lie derivative along $u^{a}$ in terms of the coordinate time derivative, and retaining only terms at second order, we have

$$
\dot{\zeta}_{i}^{(2)}=\zeta_{i}^{(2) \prime}-A \zeta_{i}^{(1) \prime}+\left(\frac{v^{j}}{a} \partial_{j} \zeta_{i}^{(1)}+\zeta_{j}^{(1)} \frac{\partial_{i} v^{j}}{a}\right)
$$

Finally, on making use of Eqs. (18), (24), and (25), and that $\Theta=3(1-A) \alpha^{\prime}$ up to first order, we can explicitly write the conservation equation (9) up to second order and on all scales:

$$
\zeta^{(2) \prime}=-\frac{H}{\bar{\rho}+\bar{P}} \Gamma^{(2)}-\frac{1}{\bar{\rho}+\bar{P}} \Gamma^{(1)} \zeta^{(1) \prime}-\frac{v^{j}}{a} \partial_{j} \zeta^{(1)} .
$$

The definition of $\Gamma^{(2)}$ can be read from the second-order expression of $\zeta^{(2)}$ by substituting $P$ to $\alpha$. For adiabatic perturbations, we find that at second order the scalar variable $\zeta$ is conserved only on large scales, when the last term on the right-hand side of Eq. (28) can be neglected.

One can extend straightforwardly the procedure presented here to higher orders in the perturbation expansion $[42,43]$. Expanding Eq. (10) at third order, after some manipulations one can write

$$
\zeta_{i}^{(3)}=\partial_{i} \zeta^{(3)}+\frac{\delta \rho}{\bar{\rho}^{\prime}} \partial_{i} \zeta^{(2)^{\prime}}+\frac{\delta \rho^{(2)}}{\bar{\rho}^{\prime}} \partial_{i} \zeta^{(1)^{\prime}}-\frac{\bar{\rho}^{\prime \prime}}{2 \bar{\rho}^{\prime 3}} \delta \rho^{2} \partial_{i} \zeta^{(1)^{\prime}}+\frac{\delta \rho^{2}}{2 \bar{\rho}^{\prime 2}} \partial_{i} \zeta^{(1)^{\prime \prime}}
$$

with

$$
\begin{aligned}
\zeta^{(3)} & =\delta \alpha^{(3)}-\frac{H}{\bar{\rho}^{\prime}} \delta \rho^{(3)}-\frac{1}{\bar{\rho}^{\prime}}\left(\delta \alpha^{\prime}-\frac{H}{\bar{\rho}^{\prime}} \delta \rho^{\prime}\right)\left(\delta \rho^{(2)}-\frac{\delta \rho \delta \rho^{\prime}}{\bar{\rho}^{\prime}}+\frac{\bar{\rho}^{\prime \prime} \delta \rho^{2}}{2 \bar{\rho}^{2} 2}\right)-\frac{\delta \rho}{\bar{\rho}^{\prime}}\left(\delta \alpha^{(2)^{\prime}}-\frac{H}{\bar{\rho}^{\prime}} \delta \rho^{(2)^{\prime}}\right) \\
& +\frac{\delta \rho^{2}}{2 \bar{\rho}^{\prime 2}}\left(\delta \alpha^{\prime \prime}-\frac{H}{\bar{\rho}^{\prime}} \delta \rho^{\prime \prime}\right)+\frac{1}{\bar{\rho}^{\prime}}\left(\frac{H}{\bar{\rho}^{\prime}}\right)^{\prime} \delta \rho^{(2)} \delta \rho-\frac{1}{\bar{\rho}^{\prime 2}}\left(\frac{H}{\bar{\rho}^{\prime}}\right)^{\prime} \delta \rho^{2} \delta \rho^{\prime}-\frac{1}{6 \bar{\rho}^{\prime}}\left[\frac{1}{\bar{\rho}^{\prime}}\left(\frac{H}{\bar{\rho}^{\prime}}\right)^{\prime}\right]^{\prime} \delta \rho^{3} .
\end{aligned}
$$

Its evolution equation up to third order can be found by expanding Eq. (9). On large scales it reads [43]

$$
\zeta^{(3) \prime} \approx-\frac{H}{\bar{\rho}+\bar{P}} \Gamma^{(3)}-\frac{1}{\bar{\rho}+\bar{P}}\left(\Gamma^{(1)} \zeta^{(2) \prime}+\Gamma^{(2)} \zeta^{(1) \prime}\right)-\frac{1}{3 H(\bar{\rho}+\bar{P})} \Gamma\left(\zeta^{\prime}\right)^{2},
$$

where $\Gamma^{(3)}$ is defined in the same way as $\zeta^{(3)}$ in Eqs. (29) and (30) with the replacements $\alpha \rightarrow P, \zeta \rightarrow \Gamma$.

In [42] one can find a discussion on perturbations beyond the third order, as well as a general expression for a quantity $\zeta^{(n)}$, defined at any order $n$, which is conserved on large scales for adiabatic perturbations.

\section{The issue of gauge-invariance}

One can check that, on large scales, $\zeta^{(2)}$ behaves as a gauge-invariant quantity. Given a vector $\xi^{a}=\sum_{n} \frac{1}{n !} \xi_{(n)}^{a}$, the gauge transformation it generates is defined by the transformation law of tensors [6] (whose coordinate functions are a particular case),

$$
\tilde{\mathbf{T}} \rightarrow e^{\mathcal{L} \xi} \mathbf{T}
$$

Since $\zeta_{a}$ vanishes at zeroth order, $\zeta_{a}$ is automatically gauge-invariant at first order, according to the first expression above. However, $\zeta_{i}$ is not gauge-invariant at second order and the corresponding gauge transformation is given by

$$
\zeta_{i}^{(2)} \rightarrow \zeta_{i}^{(2)}+\mathcal{L}_{\xi_{(1)}} \zeta_{i}^{(1)} \approx \zeta_{i}^{(2)}+\xi_{(1)}^{0} \partial_{0} \zeta_{i}^{(1)}
$$

where for the last equality we have neglected the terms of higher order in spatial gradients, concentrating on large scales. By noting that at first order $\delta \rho / \bar{\rho}^{\prime} \rightarrow \delta \rho / \bar{\rho}^{\prime}+\xi_{(1)}^{0}$, it is easy to see using Eq. (24) that $\partial_{i} \zeta^{(2)}$, or equivalently $\zeta^{(2)}$, is gauge-invariant at second order, on large scales. This can also be checked by directly applying the gauge transformation (32) on $\zeta^{(2)}$ defined in Eq. (25). This analysis can be repeated at third [43] or any higher order $n$ [42] and one can show that the quantity $\zeta^{(n)}$ is gauge invariant on large scales. 


\section{Dissipative and interacting fluids}

It is possible to extend our covariant approach to the case of dissipative and interacting fluids, as discussed in detail in [24]. In that case, the energy-momentum tensor is more complicated,

$$
T_{a b}=\rho u_{a} u_{b}+P h_{a b}+q_{a} u_{b}+q_{b} u_{a}+\pi_{a b},
$$

where the energy flow $q_{a}$ and anisotropic stress $\pi_{a b}$ satisfy

$$
q_{a} u^{a}=0, \quad \pi_{a b}=\pi_{b a}, \quad \pi_{a}^{a}=0, \quad \pi_{a b} u^{b}=0 .
$$

The continuity equation in this case reads

$$
\dot{\rho}+\Theta(\rho+P)=\mathcal{D}, \quad \mathcal{D} \equiv-\left(\pi^{a b} \sigma_{a b}+D_{a} q^{a}+2 q^{a} a_{a}\right),
$$

where $D_{a}$ denotes the spatially projected covariant derivative, orthogonal to the four-velocity $u^{a}$. For a generic tensor $\mathcal{T}$, the definition is (see e.g. [28])

$$
D_{a} \mathcal{T}_{b \ldots}{ }^{c \ldots} \equiv h_{a}{ }^{d} h_{b}{ }^{e} \ldots h_{f}^{c} \ldots \nabla_{d} \mathcal{T}_{e}{ }^{f \ldots} \ldots .
$$

As a consequence of the presence of the dissipative terms on the right-hand side of (36), the evolution equation for $\zeta_{a}$ is slightly more involved and now reads

$$
\dot{\zeta}_{a}=\frac{3 \dot{\alpha}^{2}}{\dot{\rho}}\left(\Gamma_{a}+\Sigma_{a}\right)
$$

where

$$
\Sigma_{a} \equiv D_{a} \beta-\frac{\dot{\beta}}{\dot{\rho}} D_{a} \rho, \quad \beta \equiv-\frac{\mathcal{D}}{\Theta} .
$$

This means that dissipative terms affect the evolution of the covector $\zeta_{a}$ in addition to the non-adiabatic term.

\section{COSMOLOGICAL SCALAR FIELDS}

We now consider the situation where matter is composed of several scalar fields, concentrating on the case [25]. We study $N$ scalar fields minimally coupled to gravity with Lagrangian density

$$
\mathcal{L}=-\frac{1}{2} \partial_{a} \varphi_{I} \partial^{a} \varphi^{I}-V\left(\varphi_{K}\right),
$$

where $V$ is the potential and the summation over the field indices $(I, J, \ldots)$ is implicit. For simplicity we assume here canonical kinetic terms but the nonlinear extension to a large class of models with non-canonical kinetic terms, such as those studied at the linear level in [44], has also been developed in [45]. The energy-momentum tensor derived from the above Lagrangian reads

$$
T_{a b}=\partial_{a} \varphi_{I} \partial_{b} \varphi^{I}-\frac{1}{2} g_{a b}\left(\partial_{c} \varphi_{I} \partial^{c} \varphi^{I}+2 V\right) .
$$

Given an arbitrary unit timelike vector field $u^{a}$, it is always possible to decompose this energy momentum tensor in the form (34) with, in our case,

$$
\begin{aligned}
\rho & =\frac{1}{2}\left(\dot{\varphi}_{I} \dot{\varphi}^{I}+D_{a} \varphi^{I} D^{a} \varphi_{I}\right)+V, \quad P=\frac{1}{2}\left(\dot{\varphi}_{I} \dot{\varphi}^{I}-\frac{1}{3} D_{a} \varphi_{I} D^{a} \varphi^{I}\right)-V, \\
q_{a} & =-\dot{\varphi}_{I} D_{a} \varphi^{I}, \quad \pi_{a b}=D_{a} \varphi_{I} D_{b} \varphi^{I}-\frac{1}{3} h_{a b} D_{c} \varphi_{I} D^{c} \varphi^{I} .
\end{aligned}
$$

The evolution equations for the scalar fields are the $N$ Klein-Gordon equations given by

$$
-\nabla_{a} \nabla^{a} \varphi_{I}+\frac{\partial V}{\partial \varphi_{I}}=\ddot{\varphi}_{I}+\Theta \dot{\varphi}_{I}+V_{, \varphi_{I}}-D_{a} D^{a} \varphi_{I}-a^{a} D_{a} \varphi_{I}=0
$$

where the second expression is obtained by using a decomposition into (covariant) time-like and space-like gradients defined with respect to $u^{a}$. 


\section{A. Adiabatic and entropy covectors}

For simplicity we now restrict our analysis to the case of two scalar fields, which we will denote by $\phi$ and $\chi$. In the two-field case it is possible to introduce a particular basis in the field space in which various field dependent quantities are decomposed into so-called adiabatic and entropy components. In the linear theory this decomposition was first introduced in [46] for two fields. This decomposition is particularly convenient to follow the time evolution of the curvature perturbation, which is sourced by the entropic perturbations. For the multi-field case it is discussed in [47] in the linear theory and in [48] in the nonlinear context.

In our case, the corresponding basis consists, in the two-dimensional field space, of a unit vector $e_{\sigma}^{I}$ defined in the direction of the velocity of the two fields, and thus tangent to the trajectory in field space, and of a unit vector $e_{s}^{I}$ defined along the direction orthogonal to it, namely

$$
e_{\sigma}^{I} \equiv(\dot{\phi} / \dot{\sigma}, \dot{\chi} / \dot{\sigma})=(\cos \theta, \sin \theta), \quad e_{s}^{I} \equiv(-\dot{\chi} / \dot{\sigma}, \dot{\phi} / \dot{\sigma})=(-\sin \theta, \cos \theta),
$$

where we have introduced the formal notation $\dot{\sigma} \equiv\left(\dot{\phi}^{2}+\dot{\chi}^{2}\right)^{1 / 2}$. Note that this notation can be misleading as, in general, in the nonlinear context $\dot{\sigma}$ is not the derivative along $u^{a}$ of a scalar field $\sigma$.

The angle $\theta$, which characterizes the rotation angle between the initial field basis and the adiabatic/entropic basis, is here an inhomogeneous quantity which depends on time and space. By taking the time derivative of the basis vectors $e_{\sigma}^{I}$ and $e_{s}^{I}$, we get

$$
\dot{e}_{\sigma}^{I}=\dot{\theta} e_{s}^{I}, \quad \dot{e}_{s}^{I}=-\dot{\theta} e_{\sigma}^{I}
$$

Making use of the basis (45), one can then introduce two linear combinations of the scalar field gradients and thus define two covectors, respectively denoted by $\sigma_{a}$ and $s_{a}$, as [25]

$$
\begin{aligned}
\sigma_{a} & \equiv e_{\sigma}^{I} \partial_{a} \varphi_{I}=\cos \theta \partial_{a} \phi+\sin \theta \partial_{a} \chi \\
s_{a} \equiv e_{s}^{I} \partial_{a} \varphi_{I} & =-\sin \theta \partial_{a} \phi+\cos \theta \partial_{a} \chi
\end{aligned}
$$

We will call these two covectors the adiabatic and entropy covectors, respectively, by analogy with the similar definitions in the linear context [46]. Whereas the entropy covector $s_{a}$ is orthogonal to the four-velocity $u^{a}$, i.e., $u^{a} s_{a}=0$, this is not the case for $\sigma_{a}$ which contains a "longitudinal" component: $u^{a} \sigma_{a}=\dot{\sigma}$. It is also useful to introduce the spatially projected version of (47) and (48),

$$
\sigma_{a}^{\perp} \equiv e_{\sigma}^{I} D_{a} \varphi_{I}=\sigma_{a}+\dot{\sigma} u_{a}, \quad s_{a}^{\perp} \equiv e_{s}^{I} D_{a} \varphi_{I}=s_{a} .
$$

The "adiabatic" combination of the Klein-Gordon equations, i.e., the contraction of (44) by $e_{\sigma}^{I}$, yields

$$
\ddot{\sigma}+\Theta \dot{\sigma}+V_{, \sigma}=\nabla^{a} \sigma_{a}^{\perp}-Y_{(s)},
$$

where we have defined

$$
V_{, \sigma} \equiv e_{\sigma}^{I} V_{, \varphi_{I}}, \quad Y_{(s)} \equiv \frac{1}{\dot{\sigma}}\left(\dot{s}_{a}+\dot{\theta} \sigma_{a}^{\perp}\right) s^{a}
$$

Since $e_{s}^{I} \ddot{\varphi}_{I}=\dot{\theta} \dot{\sigma}$, the "entropic" combination of the Klein-Gordon equations, i.e., the contraction of (44) with $e_{s}^{I}$, gives simply

$$
\dot{\sigma} \dot{\theta}+V_{, s}=\nabla_{a} s^{a}+Y_{(\sigma)}
$$

with

$$
V_{, s} \equiv e_{s}^{I} V_{, \varphi_{I}}, \quad Y_{(\sigma)} \equiv \frac{1}{\dot{\sigma}}\left(\dot{s}_{a}+\dot{\theta} \sigma_{a}^{\perp}\right) \sigma^{\perp}{ }^{a}
$$

In summary, the Klein-Gordon equations for the fields $\phi$ and $\chi$ have been replaced by the equations (50) and (52), whose left-hand side has exactly the same form as the homogeneous equations in a Friedmann universe. However, these equations capture the fully nonlinear dynamics of the scalar fields and their right-hand side contains nonlinear (quadratic) terms represented by $Y_{(s)}$ and $Y_{(\sigma)}$, sourcing the adiabatic and entropy equations respectively.

We now consider the evolution equations for the covectors $\sigma_{a}$ and $s_{a}$, or rather for their spatial projections. The adiabatic evolution equation is given by

$$
\begin{aligned}
& \left(\ddot{\sigma}_{a}\right)^{\perp}+\Theta\left(\dot{\sigma}_{a}\right)^{\perp}+\dot{\sigma} D_{a} \Theta+\left(V_{, \sigma \sigma}+\dot{\theta} \frac{V_{, s}}{\dot{\sigma}}\right) \sigma_{a}^{\perp}-D_{a}\left(\nabla^{c} \sigma_{c}^{\perp}\right)=\left(\dot{\theta}-\frac{V_{, s}}{\dot{\sigma}}\right) \dot{s}_{a} \\
& +\left(\ddot{\theta}-V_{, \sigma s}+\Theta \dot{\theta}\right) s_{a}-D_{a} Y_{(s)},
\end{aligned}
$$


where we have introduced the notation

$$
V_{, \sigma \sigma} \equiv e_{\sigma}^{I} e_{\sigma}^{J} V_{, \varphi_{I} \varphi_{J}}, \quad V_{, s s} \equiv e_{s}^{I} e_{s}^{J} V_{, \varphi_{I} \varphi_{J}}, \quad V_{, s \sigma} \equiv e_{s}^{I} e_{\sigma}^{J} V_{, \varphi_{I} \varphi_{J}}
$$

for the second derivatives of the potential. Note that the spatial projection and the time derivative do not commute for $\sigma_{a}$. The entropic evolution equation reads

$$
\ddot{s}_{a}-\frac{1}{\dot{\sigma}}\left(\ddot{\sigma}+V_{, \sigma}\right) \dot{s}_{a}+\left(V_{, s s}-\dot{\theta}^{2}\right) s_{a}-D_{a}\left(\nabla_{c} s^{c}\right)=-2 \dot{\theta}\left(\dot{\sigma}_{a}\right)^{\perp}+\left[\frac{\dot{\theta}}{\dot{\sigma}}\left(\ddot{\sigma}+V_{, \sigma}\right)-\ddot{\theta}-V_{, \sigma s}\right] \sigma_{a}^{\perp}+D_{a} Y_{(\sigma)}
$$

where the covectors $\dot{s}_{a}$ and $\ddot{s}_{a}$ are purely spatial, i.e. $\left(\dot{s}_{a}\right)^{\perp}=\dot{s}_{a}$ and $\left(\ddot{s}_{a}\right)^{\perp}=\ddot{s}_{a}$.

Starting from the fully nonlinear Klein-Gordon equations, we have thus managed to obtain a system of two coupled equations (54) and (56), which govern the evolution of our nonlinear adiabatic and entropy components [25]. Remarkably, they are rather simple and they look very similar to the linear equations for the perturbations $\delta \sigma$ and $\delta s$ (see [46]). Furthermore, since these equations are exact, it is straightforward to expand them up to second or higher orders, as we will show explicitly in Sec. III C.

\section{B. Generalized curvature perturbations and large-scale evolution}

Let us now turn to the generalized curvature perturbation $\zeta_{a}$, which we introduced in Eq (10) for a cosmological fluid. Although a system of several scalar fields cannot be described as a perfect fluid, its energy-momentum tensor is of the form (34) with

$$
\begin{aligned}
\rho & =\frac{1}{2}\left(\dot{\sigma}^{2}+\Pi\right)+V, \quad P=\frac{1}{2}\left(\dot{\sigma}^{2}-\frac{1}{3} \Pi\right)-V, \\
q_{a} & =-\dot{\sigma} \sigma_{a}^{\perp}, \quad \pi_{a b}=\Pi_{a b}-\frac{1}{3} h_{a b} \Pi,
\end{aligned}
$$

where we have defined

$$
\Pi_{a b} \equiv \sigma_{a}^{\perp} \sigma_{b}^{\perp}+s_{a} s_{b}, \quad \Pi \equiv \sigma_{c}^{\perp} \sigma^{\perp}{ }^{c}+s_{c} s^{c} .
$$

The quantity $\zeta_{a}$ for the two-fluid system therefore satisfies an evolution equation of the form (38).

In the linear theory, an alternative quantity to describe the primordial perturbation is the comoving curvature perturbation. A natural nonlinear extension of this quantity in our formalism is (see also [49] for an alternative definition)

$$
\mathcal{R}_{a} \equiv-D_{a} \alpha-\frac{\dot{\alpha}}{\left(\dot{\varphi}_{J} \dot{\varphi}^{J}\right)} q_{a}
$$

As in the linear case, this expression can be directly related to the analog of the Mukhanov-Sasaki variables [50, 51], if we generalize the latter by defining

$$
Q_{a}^{I} \equiv D_{a} \varphi^{I}-\frac{\dot{\varphi}^{I}}{\dot{\alpha}} D_{a} \alpha
$$

for each scalar field. The comoving curvature perturbation $\mathcal{R}_{a}$ is then proportional to the adiabatic combination of the $Q_{a}^{I}$, i.e.

$$
Q_{a} \equiv e_{\sigma I} Q_{a}^{I}=\sigma_{a}^{\perp}-\frac{\dot{\sigma}}{\dot{\alpha}} D_{a} \alpha
$$

since one can write $\mathcal{R}_{a}=\dot{\alpha} \dot{\varphi}_{I} Q_{a}^{I} /\left(\dot{\varphi}_{J} \dot{\varphi}^{J}\right)$.

Comparing the definitions of $\zeta_{a}$ and $\mathcal{R}_{a}$ shows that they satisfy the relation

$$
\zeta_{a}+\mathcal{R}_{a}=-\frac{\dot{\alpha}}{\dot{\rho}} \epsilon_{a}, \quad \epsilon_{a} \equiv D_{a} \rho-\frac{\dot{\rho}}{\dot{\sigma}} \sigma_{a}^{\perp},
$$

where the covector $\epsilon_{a}$ can be interpreted as a covariant generalization of the comoving energy density perturbation. 
Let us now concentrate on the large scale limit by neglecting terms of higher order in spatial gradients. Using the energy constraint $u^{a} G_{a b} u^{b}=8 \pi G \rho$ and the momentum constraint $u^{b} G_{b c} h_{a}^{c}=8 \pi G q_{a}$, it can be shown that the comoving energy density perturbation, $\epsilon_{a}$, defined in Eq. (63), can be neglected on large scales if the shear can also be neglected in this limit. This is the case in an expanding perturbed FLRW universe, where the shear rapidly decreases on large scales.

Then, from their definition (49), $\sigma_{a}^{\perp}$ and $s_{a}$ are first-order quantities with respect to spatial gradients while the scalars $Y_{(s)}$ and $Y_{(\sigma)}$ are second order, since they are quadratic in $\sigma_{a}^{\perp}$ and $s_{a}$ (or their time derivatives). Hence, the right-hand side of Eq. (50) and of Eq. (52) can be neglected on large scales. Taking into account these simplifications and neglecting on large scales $\epsilon_{a}$ and the terms containing $\Pi$, one finds that the evolution equations in Eqs. (54) and (56) simplify to give

$$
\left(\ddot{\sigma}_{a}\right)^{\perp}+\Theta\left(\dot{\sigma}_{a}\right)^{\perp}+\dot{\sigma} D_{a} \Theta+\left(V_{, \sigma \sigma}-\dot{\theta}^{2}\right) \sigma_{a}^{\perp} \approx 2\left(\dot{\theta} s_{a}\right)^{\cdot}-2 \dot{\theta} \frac{V_{, \sigma}}{\dot{\sigma}} s_{a}
$$

and

$$
\ddot{s}_{a}+\Theta \dot{s}_{a}+\left(V_{, s s}+3 \dot{\theta}^{2}\right) s_{a} \approx 0 .
$$

While the entropy mode appears as a source term in the adiabatic equation, the entropy equation involves only the covector $s_{a}$.

On large scales, neglecting $\epsilon_{a}$ in Eq. (63) implies that the uniform density and comoving perturbations $\zeta_{a}$ and $\mathcal{R}_{a}$ coincide (up to a sign),

$$
\zeta_{a}+\mathcal{R}_{a} \approx 0
$$

Moreover, in the same limit, the source term in the evolution equation for $\zeta_{a}$, Eq. (38), is dominated by the gradient of the potential $V$ along the entropic direction, so that

$$
\dot{\zeta}_{a} \approx-\frac{2}{3} \frac{\Theta^{2}}{\dot{\rho}} V_{, s} s_{a}
$$

This equation is sufficient to describe the evolution of the adiabatic mode in the large scale limit, making Eq. (64) redundant. The analogs of the spatial components of Eqs. (67) and (65) have also been derived in [48].

\section{Link with the coordinate approach}

\section{Linear theory}

We now relate our covariant approach with the more familiar coordinate based formalism, starting with the linear theory. We use the metric and notation of Sec. II B 1 and the scalar fields are decomposed into a background and a perturbed part, $\varphi_{I}\left(t, x^{i}\right)=\bar{\varphi}_{I}(t)+\delta \varphi_{I}\left(t, x^{i}\right)$. We now need to specify the components of the unit vector $u^{a}$, which defines the time direction in our covariant approach. In contrast with the perfect fluid case, where $u^{a}$ has a physical meaning, the unit vector $u^{a}$ is here arbitrary. In a given coordinate system it is convenient to choose $u^{\mu}$ such that $u_{i}=0$ at first order. This implies that the components of $u^{\mu}$ and of the "acceleration" vector are given respectively by

$$
u^{\mu}=\left\{1-A,-B^{i} / a\right\}, \quad a^{\mu}=\left\{0, \vec{\nabla}^{i} A / a^{2}\right\} .
$$

The background equations of motion can be deduced immediately from Eqs. (50) and (52) and read

$$
\bar{\sigma}^{\prime \prime}+3 H \bar{\sigma}^{\prime}+\bar{V}_{, \sigma}=0, \quad \bar{\sigma}^{\prime} \bar{\theta}^{\prime}+\bar{V}_{, s}=0 .
$$

From their definition, one finds that the spatial components of $\sigma_{a}$ and $s_{a}$, at linear order, can be expressed as

$$
\begin{aligned}
\delta \sigma_{i}=\partial_{i} \delta \sigma, & \delta \sigma \equiv \cos \bar{\theta} \delta \phi+\sin \bar{\theta} \delta \chi \\
\delta s_{i}=\partial_{i} \delta s, & \delta s \equiv \cos \bar{\theta} \delta \chi-\sin \bar{\theta} \delta \phi
\end{aligned}
$$

which coincide with the notations of [46]. 
Linearizing the evolution equations for $\sigma_{a}$ and $s_{a}$, eqs. (54) and (56), one easily obtains the linearized equations for $\delta \sigma$ and $\delta s$. The latter can be written as

$$
\delta s^{\prime \prime}+3 H \delta s^{\prime}+\left(\bar{V}_{, s s}+3 \bar{\theta}^{\prime 2}\right) \delta s-\frac{1}{a^{2}} \vec{\nabla}^{2} \delta s=-2 \frac{\bar{\theta}^{\prime}}{\bar{\sigma}^{\prime}} \delta \epsilon,
$$

where on the right-hand side appears the first-order comoving energy density perturbation $\delta \epsilon$, defined by

$$
\delta \epsilon_{i}=\partial_{i} \delta \epsilon, \quad \delta \epsilon \equiv \delta \rho-\frac{\bar{\rho}^{\prime}}{\bar{\sigma}^{\prime}} \delta \sigma,
$$

which follows from the definition (63) of $\epsilon_{a}$. The quantity $\delta \epsilon$ can in general be neglected on large scales, since it is second order in spatial gradients, as can be seen from the relativistic Poisson-like equation.

Let us now turn to the adiabatic equation. Since $\delta \sigma$ is not gauge-invariant, in contrast with $\delta s$, it is more useful to consider the gauge invariant Sasaki-Mukhanov variable $Q_{\mathrm{SM}}$, defined as $[50,51]$

$$
Q_{\mathrm{SM}} \equiv \delta \sigma+\frac{\bar{\sigma}^{\prime}}{H} \psi
$$

Note that the above traditional definition coincides with the scalar quantity $Q$ that can be extracted from our definition of $Q_{a}$ given earlier in Eq. (62), via $Q_{i}=\partial_{i} Q$, only in the large scale limit, because $\psi$ and $-\delta \alpha$ coincide only in this limit. The evolution equation of $Q_{\mathrm{SM}}$ reads $[46,52]$

$$
Q_{\mathrm{SM}}^{\prime \prime}+3 H Q_{\mathrm{SM}}^{\prime}+\left[\bar{V}_{, \sigma \sigma}-\bar{\theta}^{\prime 2}-2 \frac{H^{\prime}}{H}\left(\frac{\bar{V}_{, \sigma}}{\bar{\sigma}^{\prime}}+\frac{H^{\prime}}{H}-\frac{\bar{\sigma}^{\prime \prime}}{\bar{\sigma}^{\prime}}\right)-\frac{\vec{\nabla}^{2}}{a^{2}}\right] Q_{\mathrm{SM}}=2\left(\bar{\theta}^{\prime} \delta s\right)^{\prime}-2 \bar{\theta}^{\prime}\left(\frac{\bar{V}_{, \sigma}}{\bar{\sigma}^{\prime}}+\frac{H^{\prime}}{H}\right) \delta s .
$$

On large scales the adiabatic evolution is simpler and governed by the first integral

$$
Q_{\mathrm{SM}}^{\prime}+\left(\frac{H^{\prime}}{H}-\frac{\sigma^{\prime \prime}}{\sigma^{\prime}}\right) Q_{\mathrm{SM}}-2 \bar{\theta}^{\prime} \delta s \approx 0
$$

which simply expresses that $\delta \epsilon$, written in terms of $Q_{\mathrm{SM}}$ and $\delta s$, is negligible on large scales. One can easily check that the large-scale limit of (75) follows from the first integral (76).

Let us now consider the evolution equation for $\zeta_{a}$. The spatial components of $\zeta_{a}$, at linear order, are given by eq. (18) as for the fluid case: $\zeta_{i} \equiv \partial_{i} \zeta$. Similarly, one can define $\mathcal{R}$ by $\mathcal{R}_{i}=\partial_{i} \mathcal{R}$. Note that $\mathcal{R}$ coincides with the familiar comoving curvature perturbation only in the large scale limit, because of the difference between $\alpha$ and $\psi$, as already discussed for $\zeta$. Eq. (66) implies a simple relation between $\zeta$ and $\mathcal{R}$,

$$
\zeta+\mathcal{R}=-\frac{\bar{\alpha}^{\prime}}{\bar{\rho}^{\prime}} \delta \epsilon
$$

which shows that $\zeta$ and $-\mathcal{R}$ coincide on large scales.

The evolution equation for $\zeta$ on large scales follows from eq. (67) which, after linearization, yields

$$
\zeta^{\prime} \approx-\frac{2 H}{\bar{\sigma}^{\prime}} \bar{\theta}^{\prime} \delta s .
$$

One thus recovers the familiar linear result [46] that the entropy perturbation is sourcing the evolution of the uniform density curvature perturbation on large scales.

\section{Second-order perturbations}

As for the perfect fluid case in section II B 2, we now expand the equations governing $\sigma_{a}, s_{a}$ and $\zeta_{a}$ at second order in the perturbations. For simplicity, we will restrict ourselves to large scales and we will thus start from the equations expanded in spatial gradients discussed in Sec. III B.

The second-order evolution equations for the perturbations of a single scalar field, in a coordinate based approach, has been considered in several references (see for instance $[11,12,53,54]$ ). The multi-field case has been first studied by Malik in $[55,56]$ and, using the separate universe approach, in $[57,58]$. Here, we obtain directly the second-order evolution equations in terms of the adiabatic and entropic perturbations. 
By expanding Eq. (47) and Eq. (48) up to second order, one can write [25]

$$
\begin{aligned}
\delta \sigma_{i}^{(2)} & =\partial_{i} \delta \sigma^{(2)}+\frac{\bar{\theta}^{\prime}}{\bar{\sigma}^{\prime}} \delta \sigma \partial_{i} \delta s-\frac{1}{\bar{\sigma}^{\prime}} V_{i}, \\
\delta s_{i}^{(2)} & =\partial_{i} \delta s^{(2)}+\frac{\delta \sigma}{\bar{\sigma}^{\prime}} \partial_{i} \delta s^{\prime},
\end{aligned}
$$

with

$$
\begin{aligned}
\delta \sigma^{(2)} & \equiv \frac{\bar{\phi}^{\prime}}{\bar{\sigma}^{\prime}} \delta \phi^{(2)}+\frac{\bar{\chi}^{\prime}}{\bar{\sigma}^{\prime}} \delta \chi^{(2)}+\frac{1}{2 \bar{\sigma}^{\prime}} \delta s \delta s^{\prime}, \\
\delta s^{(2)} & \equiv-\frac{\bar{\chi}^{\prime}}{\bar{\sigma}^{\prime}} \delta \phi^{(2)}+\frac{\bar{\phi}^{\prime}}{\bar{\sigma}^{\prime}} \delta \chi^{(2)}-\frac{\delta \sigma}{\bar{\sigma}^{\prime}}\left(\delta s^{\prime}+\frac{\bar{\theta}^{\prime}}{2} \delta \sigma\right),
\end{aligned}
$$

and where we have defined the spatial vector

$$
V_{i} \equiv \frac{1}{2}\left(\delta s \partial_{i} \delta s^{\prime}-\delta s^{\prime} \partial_{i} \delta s\right)
$$

The definition of $\delta s^{(2)}$ is chosen such that it is gauge invariant on large scales. Since the adiabatic component $\sigma_{a}$ does not vanish at zeroth order, $\delta \sigma^{(2)}$ is not a gauge invariant variable and can be chosen for convenience. Its definition here is such that the momentum perturbation almost vanishes (up to $V_{i}$ ) when $\delta \sigma^{(2)}=0$.

The presence of the spatial vector $V_{i}$ on the right-hand side of Eq. (79) is due to the fact that $\sigma_{a}$ defined in Eq. (47) is not hypersurface orthogonal. Indeed, one can check using its definition that $\sigma_{[a} \nabla_{b} \sigma_{c]}=\dot{\sigma}^{-1} \sigma_{[a} \dot{s}_{b} s_{c]}$. As the momentum $\delta q_{i}$ is proportional to $\delta \sigma_{i}^{\perp}$, Eq. (58), this implies that if $V_{i}$ does not vanish one cannot define at second-order a comoving gauge, i.e., such that $\delta q_{i}^{(1)}=0$ and $\delta q_{i}^{(2)}=0$, in contrast with the linear theory or the single-field case [48]. However, the evolution equation for $V_{i}$ on large scales reads

$$
V_{i}^{\prime}+3 H V_{i} \approx 0,
$$

which implies that in an expanding universe $V_{i}$ decays as $a^{-3}$ and rapidly become negligible even if it is nonzero initially. Consequently, in the following we will ignore $V_{i}$ on large scales.

Since $\delta \sigma^{(2)}$ is not gauge-invariant, it is useful to consider the Sasaki-Mukhanov variable at second order $Q_{\mathrm{SM}}^{(2)}$, which on large scales coincides with the scalar quantity $Q^{(2)}$ which can be extracted by expanding at second order $Q_{a}$ defined in Eq. (62),

$$
Q_{i}^{(2)}=\partial_{i} Q^{(2)}+\frac{\delta \alpha}{H} \partial_{i} Q^{\prime}+\frac{\bar{\theta}^{\prime}}{\bar{\sigma}^{\prime}} Q \partial_{i} \delta s-\frac{1}{\bar{\sigma}^{\prime}} V_{i}
$$

The Sasaki-Mukhanov $Q_{\mathrm{SM}}^{(2)}$ can be found by replacing Eq. (26) in the definition of $Q^{(2)}$, which yields

$$
Q_{\mathrm{SM}}^{(2)} \equiv \delta \sigma^{(2)}+\frac{\bar{\sigma}^{\prime}}{H}\left(\psi^{(2)}+\psi^{2}\right)+\frac{\psi}{H}\left[Q_{\mathrm{SM}}^{(1) \prime}-\frac{1}{2}\left(\frac{\bar{\sigma}^{\prime}}{H}\right)^{\prime} \psi-\bar{\theta}^{\prime} \delta s\right] .
$$

Restricted to a single scalar field, this definition coincides with the one given in [55]. By using the energy and momentum constraint equations at second order it is possible to derive a first integral for $Q_{\mathrm{SM}}^{(2)}[25]$, extending at second order Eq. (76).

Here we will simply derive the large-scale evolution equation for $\zeta$ at second order, which is enough to describe the evolution of the adiabatic mode. Expanding up to second order Eq. (67) yields

$$
\zeta^{(2) \prime} \approx-\frac{H}{\bar{\sigma}^{\prime 2}}\left[2 \bar{\theta}^{\prime} \bar{\sigma}^{\prime} \delta s^{(2)}-\left(\bar{V}_{, s s}+4 \bar{\theta}^{\prime 2}\right) \delta s^{2}+\frac{\bar{V}_{, \sigma}}{\bar{\sigma}^{\prime}} \delta s \delta s^{\prime}\right] .
$$

It is also useful to express our results in terms of $\mathcal{R}_{a}$. The spatial components of $\mathcal{R}_{a}$ can be decomposed as

$$
\mathcal{R}_{i}^{(2)}=\partial_{i} \mathcal{R}^{(2)}+\frac{\delta \sigma}{\bar{\sigma}^{\prime}} \partial_{i} \mathcal{R}^{(1) \prime}-\frac{H}{\bar{\sigma}^{\prime 2}} V_{i},
$$

with

$$
\mathcal{R}^{(2)} \equiv-\delta \alpha^{(2)}+\frac{H}{\bar{\sigma}^{\prime}} \delta \sigma^{(2)}+\frac{\delta \sigma}{\bar{\sigma}^{\prime}}\left[-\mathcal{R}^{(1) \prime}+\frac{1}{2}\left(\frac{H}{\bar{\sigma}^{\prime}}\right)^{\prime} \delta \sigma+\bar{\theta}^{\prime} \frac{H}{\bar{\sigma}^{\prime}} \delta s\right] .
$$


The last term in Eq. (88) comes from the fact that, in contrast to $\zeta_{a}, \mathcal{R}_{a}$ is defined in terms of the spatial momentum which cannot be expressed in general as a pure gradient. However, in an expanding universe this term can be neglected and $\mathcal{R}^{(2)}$ coincides with the second-order comoving curvature perturbation defined in [54, 59].

It is easy to derive a first-order (in time) evolution equation for $\mathcal{R}^{(2)}$ by noting that $\zeta^{(2)}$ and $\mathcal{R}^{(2)}$ are related on large scales. Indeed, by using Eqs. (24) and (88), neglecting $\delta \epsilon^{(1)}$ and $V_{i}$, and making use of $\zeta^{(1)}+\mathcal{R}^{(1)} \approx 0$, one can show that the spatial component of Eq. (66) yields $\zeta^{(2)}+\mathcal{R}^{(2)} \approx 0$ [54]. From this relation and the second-order evolution equation of $\zeta$, Eq. (87), one can find a large-scale evolution equation for $\mathcal{R}$ at second order,

$$
\mathcal{R}^{(2) \prime} \approx \frac{H}{\bar{\sigma}^{\prime 2}}\left[2 \bar{\theta}^{\prime} \bar{\sigma}^{\prime} \delta s^{(2)}-\left(\bar{V}_{, s s}+4 \bar{\theta}^{\prime 2}\right) \delta s^{2}+\frac{\bar{V}_{, \sigma}}{\bar{\sigma}^{\prime}} \delta s \delta s^{\prime}\right] .
$$

The second-order perturbation $\mathcal{R}^{(2)}$ can be related on large scales to $Q_{\mathrm{SM}}^{(2)}$ by combining Eqs. (86) and (89) and using Eq. (26). One obtains

$$
\mathcal{R}^{(2)} \approx \frac{H}{\bar{\sigma}^{\prime}}\left[Q_{\mathrm{SM}}^{(2)}-\frac{1}{\bar{\sigma}^{\prime}}\left(Q_{\mathrm{SM}}^{\prime}-\bar{\theta}^{\prime} \delta s\right) Q_{\mathrm{SM}}-\frac{1}{2 H}\left(\frac{H}{\bar{\sigma}^{\prime}}\right)^{\prime} Q_{\mathrm{SM}}^{2}\right],
$$

which can be used to show that Eq. (90) can be rewritten as a first integral for $Q_{\mathrm{SM}}^{(2)}$.

Let us now discuss the second order evolution of the entropy perturbation $\delta s$. On large scales this is obtained by simply expanding the spatial components of Eq. (65) up to second order, which gives

$$
\delta s^{(2) \prime \prime}+3 H \delta s^{(2) \prime}+\left(\bar{V}_{, s s}+3 \bar{\theta}^{\prime 2}\right) \delta s^{(2)} \approx-\frac{\bar{\theta}^{\prime}}{\bar{\sigma}^{\prime}} \delta s^{\prime 2}-\frac{2}{\bar{\sigma}^{\prime}}\left(\bar{\theta}^{\prime \prime}+\bar{\theta}^{\prime} \frac{\bar{V}_{, \sigma}}{\bar{\sigma}^{\prime}}-\frac{3}{2} H \bar{\theta}^{\prime}\right) \delta s \delta s^{\prime}-\left(\frac{1}{2} \bar{V}_{, s s s}-5 \frac{\bar{\theta}^{\prime}}{\bar{\sigma}^{\prime}} \bar{V}_{, s s}-9 \frac{\bar{\theta}^{\prime 3}}{\bar{\sigma}^{\prime}}\right) \delta s^{2} .
$$

As in the linear theory, the entropy perturbation evolves independently of the adiabatic component on large scales.

\section{Third-order perturbations}

It is straightforward to extend the same procedure at third order, as discussed in detail in [43]. The evolution equation for $\zeta^{(3)}$ on large scales can be obtained by expanding up to third order Eq. (67), which yields an equation of the form

$$
\zeta^{(3) \prime} \approx \mathcal{S}_{\zeta}^{(3)}\left[\delta s, \delta s^{\prime}, \delta s^{(2)}, \delta s^{(2) \prime}\right],
$$

where the source term on the right-hand side contains terms cubic in first order perturbations $\left(\delta s\right.$ or $\left.\delta s^{\prime}\right)$ and terms that are products of second order perturbations $\left(\delta s^{(2)}\right.$ or $\left.\delta s^{(2) \prime}\right)$ with first order perturbations $\left(\delta s\right.$ or $\left.\delta s^{\prime}\right)$. The explicit definition of $\delta s^{(3)}$ used in this expansion is

$$
\delta s^{(3)} \equiv \bar{e}_{s I} \delta \phi^{I(3)}-\frac{\delta \sigma^{(2)}}{\bar{\sigma}^{\prime}}\left(\delta s^{\prime}+\bar{\theta}^{\prime} \delta \sigma\right)-\frac{\delta \sigma}{\bar{\sigma}^{\prime}} \delta s^{(2)^{\prime}}-\frac{\delta \sigma^{2}}{2 \bar{\sigma}^{\prime 2}}\left(\delta s^{\prime \prime}-\frac{\bar{\sigma}^{\prime \prime}}{\bar{\sigma}} \delta s^{\prime}+\bar{\theta}^{\prime} 2 \delta s\right)-\frac{\delta \sigma^{3}}{6 \bar{\sigma}^{\prime}}\left(\frac{\bar{\theta}^{\prime}}{\bar{\sigma}^{\prime}}\right)^{\prime}-\frac{\bar{\theta}^{\prime}}{2 \bar{\sigma}^{\prime 2}} \delta s \delta s^{\prime} \delta \sigma,
$$

which can be determined by an appropriate expansion of $s_{a}$ at third order, similar to the second order expansion (80).

To complete this equation, one needs to derive the second order (in time) equation of motion at third order on large scales. This is done by expanding Eq. (65) up to third order, which yields an equation of the form

$$
\delta s^{(3)^{\prime \prime}}+3 H \delta s^{(3)^{\prime}}+\left(\bar{V}_{, s s}+3 \bar{\theta}^{\prime 2}\right) \delta s^{(3)} \approx \mathcal{S}_{s}^{(3)}\left[\delta s, \delta s^{\prime}, \delta s^{(2)}, \delta s^{(2) \prime}\right] .
$$

The explicit expressions of the source terms $\mathcal{S}_{\zeta}$ and $\mathcal{S}_{s}$, which have been derived explicitly in [43], are given in the Appendix.

\section{An application: super-Hubble generation of non-Gaussian perturbations}

An important application of the above formalism is the computation of the non-Gaussianities of the primordial curvature perturbation. The computation of $\zeta$ and $\delta s$, by integration of their respective evolution equations up to the required order, can be seen as an alternative to the $\delta N$ formalism, described in the article by Wands in this 
issue [60]. It is especially useful when the background evolution is complicated so that one cannot express analytically the number of e-folds as a function of the initial scalar field amplitudes.

Formally, one can write the solutions of the evolution equations as

$$
\begin{aligned}
\zeta & =\zeta_{*}+T_{\zeta}^{(1)} \delta s_{*}+T_{\zeta}^{(2)} \delta s_{*}^{2}+T_{\zeta}^{(3)} \delta s_{*}^{3}+\ldots, \\
\delta s & =T_{s}^{(1)} \delta s_{*}+T_{s}^{(2)} \delta s_{*}^{2}+T_{s}^{(3)} \delta s_{*}^{3}+\ldots
\end{aligned}
$$

where the $T_{\zeta}^{(n)}$ and $T_{s}^{(n)}$ correspond to transfer functions, for $\zeta$ and $\delta s$, respectively, at the $n$-th order and $\zeta_{*}, \delta s_{*}$ are their initial conditions at Hubble exit. In particular, we are interested in the values of $\zeta$ and $\delta s$ after inflation, at the onset of the radiation dominated era.

Neglecting slow-roll corrections, the field perturbations $Q_{\mathrm{SM} *}$ and $\delta s_{*}$ at Hubble crossing can be treated as Gaussian, with respective power spectra $P_{Q_{*}}(k)$ and $P_{\delta s_{*}}(k)$ (see Appendix of [61] for an explicit calculation of their 3-point functions). Note that there exist models with non-standard kinetic terms where the perturbations just after horizon crossing are non-Gaussian (see [7] and the article of K. Koyama in this issue [62] for a general discussion on multi-field models of this type) and where the entropy perturbations can play a crucial rôle [63]. However, we will not consider these models here.

The perturbations $Q_{\mathrm{SM} *}$ and $\delta s_{*}$ are also independent random fields, at least at leading order in slow-roll [64, 65]. Moreover, although the relation between $\zeta$ and $Q_{\mathrm{SM}}$ is in principle nonlinear (as illustrated for example by Eq. (91) for $\mathcal{R}$ which coincides with $\zeta$ on large scales), at horizon crossing the linear relation dominates in slow-roll models so that $\zeta_{*} \simeq-\left(H / \bar{\sigma}^{\prime}\right) Q_{\mathrm{SM} *}[61]$.

\section{Curvature perturbations}

Using Eqs. (96) and (97) one finds that the final 2-point, 3-point and 4-point functions of $\zeta$, which are in principle observable, are given respectively by

$$
\begin{aligned}
\left\langle\zeta_{\vec{k}} \zeta_{\vec{k}^{\prime}}\right\rangle= & (2 \pi)^{3} \delta\left(\vec{k}+\vec{k}^{\prime}\right) P_{\zeta}(k), \quad P_{\zeta}(k) \equiv\left(2 \epsilon_{*} M_{P}^{2}\right)^{-1} P_{Q_{*}}(k)+\left(T_{\zeta}^{(1)}\right)^{2} P_{\delta s_{*}}(k), \\
\left\langle\zeta_{\vec{k}_{1}} \zeta_{\vec{k}_{2}} \zeta_{\vec{k}_{3}}\right\rangle \simeq & 2\left(T_{\zeta}^{(1)}\right)^{2} T_{\zeta}^{(2)}\left[P_{\delta s_{*}}\left(k_{1}\right) P_{\delta s_{*}}\left(k_{2}\right)+2 \text { cyclic }\right] \\
\left\langle\zeta_{\vec{k}_{1}} \zeta_{\vec{k}_{2}} \zeta_{\vec{k}_{3}} \zeta_{\vec{k}_{4}}\right\rangle \simeq & 4\left(T_{\zeta}^{(1)}\right)^{2}\left(T_{\zeta}^{(2)}\right)^{2}\left[P_{\delta s_{*}}\left(k_{1}\right) P_{\delta s_{*}}\left(k_{2}\right) P_{\delta s_{*}}\left(k_{13}\right)+11 \text { perms }\right] \\
& +6\left(T_{\zeta}^{(1)}\right)^{3} T_{\zeta}^{(3)}\left[P_{\delta s_{*}}\left(k_{1}\right) P_{\delta s_{*}}\left(k_{2}\right) P_{\delta s_{*}}\left(k_{3}\right)+3 \text { cyclic }\right],
\end{aligned}
$$

where we have introduced the slow-roll parameter $\epsilon \equiv 4 \pi G \bar{\sigma}^{2} / H^{2}$ and $M_{P} \equiv(8 \pi G)^{-1 / 2}$.

If the power spectrum is dominated by its entropy contribution, i.e. $\left(T_{\zeta}^{(1)}\right)^{2} P_{\delta s_{*}} \gg\left(2 \epsilon_{*} M_{P}^{2}\right)^{-1} P_{Q_{*}}$, then one can easily relate the non-Gaussianities generated by the isocurvature field to the local nonlinear parameters $f_{\mathrm{NL}}, \tau_{\mathrm{NL}}$ and $g_{\mathrm{NL}}$, defined by $[57,59,66,67]$

$$
\begin{gathered}
\left\langle\zeta_{\vec{k}_{1}} \zeta_{\vec{k}_{2}} \zeta_{\vec{k}_{3}}\right\rangle=\frac{6}{5} f_{\mathrm{NL}}\left[P_{\zeta}\left(k_{1}\right) P_{\zeta}\left(k_{2}\right)+2 \text { cyclic }\right] \\
\left\langle\zeta_{\vec{k}_{1}} \zeta_{\vec{k}_{2}} \zeta_{\vec{k}_{3}} \zeta_{\vec{k}_{4}}\right\rangle=\tau_{\mathrm{NL}}\left[P_{\zeta}\left(k_{1}\right) P_{\zeta}\left(k_{2}\right) P_{\zeta}\left(k_{13}\right)+11 \text { perms }\right]+\frac{54}{25} g_{\mathrm{NL}}\left[P_{\zeta}\left(k_{1}\right) P_{\zeta}\left(k_{2}\right) P_{\zeta}\left(k_{3}\right)+3 \text { cyclic }\right] .
\end{gathered}
$$

From Eqs. (99) and (100) one finds

$$
f_{\mathrm{NL}}=\frac{5}{3} \frac{T_{\zeta}^{(2)}}{\left(T_{\zeta}^{(1)}\right)^{2}}, \quad \tau_{\mathrm{NL}}=4 \frac{\left(T_{\zeta}^{(2)}\right)^{2}}{\left(T_{\zeta}^{(1)}\right)^{4}}, \quad g_{\mathrm{NL}}=\frac{25}{9} \frac{T_{\zeta}^{(3)}}{\left(T_{\zeta}^{(1)}\right)^{3}} .
$$

These expressions can be applied to compute the non-Gaussianities generated in multifield models. For instance, in the case of two fields, for separable potentials - potentials that can be written as the sum or product of two functions dependent only on one of the fields, the transfer functions can be computed analytically either from the evolution equations for adiabatic and isocurvature perturbations derived in this section or by using the $\delta N$-formalism $[58,68,69]$. It has been shown that the non-Gaussianity generated after inflation are slow-roll suppressed in these cases (see also the review by Byrnes and Choi [70]). The non-linear evolution of the isocurvature field during inflation can generate a large non-vanishing 3 and 4-point functions [71, 72], as reviewed by Bernardeau in this issue [73]. From Eq. (103), the conditions to obtain large nonlinear parameters are $T_{\zeta}^{(2)} \gg\left(T_{\zeta}^{(1)}\right)^{2}$ and $T_{\zeta}^{(3)} \gg\left(T_{\zeta}^{(1)}\right)^{3}$. 
Eqs. (103), together with the evolution equations for adiabatic and isocurvature perturbations derived in this section, have also been applied to compute the non-Gaussianities generated during the new Ekpyrotic scenario [43, 74-76] with results that agree with those obtained using the $\delta N$-formalism $[43,77]$.

A similar approach (although keeping the original scalar fields instead of a decomposition into adiabatic and entropic modes) has been used to compute the evolution of $\zeta$ up to second order, and thus obtain the non-Gaussianities, in the context of hybrid inflation [78-81]. In these works the curvature perturbation at second order was expressed as a time integral of an expression quadractic in the linear perturbations of the tachyonic field. The same procedure can also been used to study the preheating phase at the end of hybrid inflation [80, 81].

\section{Isocurvature perturbations}

It is possible, although not necessary, that the entropy perturbations generated during inflation survive after inflation, in the form of traditional entropy or isocurvature perturbations, i.e. fluctuations of the relative number of particles between two species (for instance, the number of photons per cold dark matter particle). In this case, the accessible cosmological data would result from a combination of curvature and isocurvature perturbations, which could be correlated [82]. So far, there has been no detection of an isocurvature perturbation in the CMB data and the constraints on the power spectra are already tight [83]. One could also envisage that an isocurvature component might be detectable through its own higher-order correlations or its higher-order correlations with the adiabatic component, as discussed in [61, 84-87]. Such a possibility can arise for instance in the mixed curvaton and inflaton scenario introduced in [88].

In the radiation dominated era the adiabatic perturbation coincides with the perturbation in the radiation fluid which, using Eq. (12) with $w=1 / 3$, can be written as

$$
\zeta_{a}^{\mathrm{r}}=\partial_{a} \zeta, \quad \zeta \equiv \delta \alpha+\frac{1}{4} \ln \left(\frac{\rho_{\mathrm{r}}}{\bar{\rho}_{\mathrm{r}}}\right) .
$$

The isocurvature perturbation between the cold dark matter and radiation, say, can be defined as

$$
S_{a}=3\left(\zeta_{a}^{\mathrm{c}}-\zeta_{a}^{\mathrm{r}}\right)=\partial_{a} S, \quad S \equiv \ln \left(\frac{\rho_{\mathrm{c}}}{\bar{\rho}_{\mathrm{c}}}\right)-\frac{3}{4} \ln \left(\frac{\rho_{\mathrm{r}}}{\bar{\rho}_{\mathrm{r}}}\right) .
$$

Formally, one can write the relation between the entropy perturbation during inflation and the final isocurvature perturbation in the form

$$
S=T_{S}^{(1)} \delta s_{*}+T_{S}^{(2)} \delta s_{*}^{2}+T_{S}^{(3)} \delta s_{*}^{3}+\ldots
$$

where the transfer functions $T_{S}^{(n)}$ depend on the details of the model. Analytically solvable examples of $T_{S}^{(2)}$ are given for instance in [61].

The observational upper limit on the fraction of isocurvature fluctuations allowed by data (see [83]) implies that the linear transfer coefficient $T_{S}^{(1)}$ must be very small. Interestingly, however, $T_{S}^{(2)}$ and $T_{S}^{(3)}$ could still be large and the non-adiabatic perturbation detectable through its effect on higher-order correlation functions. Indeed, neglecting $T_{S}^{(1)}$, one finds that the non-vanishing 3 and 4-point correlation functions are given by

$$
\begin{gathered}
\left\langle\zeta_{\vec{k}_{1}} \zeta_{\vec{k}_{2}} S_{\vec{k}_{3}}\right\rangle \simeq 2\left(T_{\zeta}^{(1)}\right)^{2} T_{S}^{(2)} P_{\delta s_{*}}\left(k_{1}\right) P_{\delta s_{*}}\left(k_{2}\right) \\
\left\langle\zeta_{\vec{k}_{1}} \zeta_{\vec{k}_{2}} S_{\vec{k}_{3}} S_{\vec{k}_{4}}\right\rangle \simeq 4\left(T_{\zeta}^{(1)}\right)^{2}\left(T_{S}^{(2)}\right)^{2} P_{\delta s_{*}}\left(k_{1}\right) P_{\delta s_{*}}\left(k_{2}\right)\left[P_{\delta s_{*}}\left(k_{13}\right)+P_{\delta s_{*}}\left(k_{23}\right)\right] \\
\left\langle\zeta_{\vec{k}_{1}} \zeta_{\vec{k}_{2}} \zeta_{\vec{k}_{3}} S_{\vec{k}_{4}}\right\rangle \simeq 4\left(T_{\zeta}^{(1)}\right)^{2} T_{\zeta}^{(2)} T_{S}^{(2)}\left\{P_{\delta s_{*}}\left(k_{1}\right) P_{\delta s_{*}}\left(k_{2}\right)\left[P_{\delta s_{*}}\left(k_{13}\right)+P_{\delta s_{*}}\left(k_{23}\right)\right]+2 \operatorname{cyclic}(1,2,3)\right\} \\
+6\left(T_{\zeta}^{(1)}\right)^{3} T_{S}^{(3)} P_{\delta s_{*}}\left(k_{1}\right) P_{\delta s_{*}}\left(k_{2}\right) P_{\delta s_{*}}\left(k_{3}\right) .
\end{gathered}
$$

It would thus be interesting to obtain some observational constraints on the isocurvature non-Gaussianities from the future data, such as those collected by the Planck satellite. 


\section{Appendix: source terms at third order}

The source terms that appear on the right hand side of (93) and (95) are respectively given by [43]

$$
\begin{aligned}
\mathcal{S}_{\zeta}^{(3)}\left[\delta s, \delta s^{\prime}, \delta s^{(2)}, \delta s^{(2) \prime}\right] \equiv & -\frac{H}{\bar{\sigma}^{\prime 2}}\left[2 \bar{\theta}^{\prime} \bar{\sigma}^{\prime} \delta s^{(3)}-2\left(\bar{V}_{, s s}+4 \bar{\theta}^{\prime 2}\right) \delta s \delta s^{(2)}+\frac{\bar{V}_{, \sigma}}{\bar{\sigma}^{\prime}}\left(\delta s^{(2)} \delta s^{\prime}\right)^{\prime}\right. \\
& \left.+\left(\frac{\bar{V}_{, s \sigma}}{\bar{\sigma}^{\prime}}-\frac{11}{3} \frac{\bar{\theta}^{\prime} \bar{V}_{, \sigma}}{\bar{\sigma}^{\prime 2}}\right) \delta s^{2} \delta s^{\prime}+\left(8 \frac{\bar{\theta}^{\prime 3}}{\bar{\sigma}^{\prime}}+4 \frac{\bar{\theta}^{\prime}}{\bar{\sigma}^{\prime}} \bar{V}_{, s s}-\frac{1}{3} \bar{V}_{, s s s}\right) \delta s^{3}\right] .
\end{aligned}
$$

and

$$
\begin{aligned}
& \mathcal{S}_{s}^{(3)}\left[\delta s, \delta s^{\prime}, \delta s^{(2)}, \delta s^{(2) \prime}\right]=-2 \frac{\bar{\theta}^{\prime}}{\bar{\sigma}^{\prime}} \delta s^{\prime} \delta s^{(2) \prime} \\
& -\frac{2}{\bar{\sigma}^{\prime}}\left(\bar{\theta}^{\prime \prime}+\bar{\theta}^{\prime} \frac{\bar{V}_{, \sigma}}{\bar{\sigma}^{\prime}}-\frac{3}{2} H \bar{\theta}^{\prime}\right)\left(\delta s \delta s^{(2)}\right)^{\prime}-\left(\bar{V}_{, s s s}-10 \bar{\theta}^{\prime} \frac{\bar{V}_{, s s}}{\bar{\sigma}^{\prime}}-18 \frac{\bar{\theta}^{\prime 3}}{\bar{\sigma}^{\prime}}\right) \delta s \delta s^{(2)} \\
& -\frac{\bar{V}_{, \sigma}}{\bar{\sigma}^{\prime 3}} \delta s^{\prime 3}-\left(\frac{\bar{V}_{, \sigma \sigma}}{\bar{\sigma}^{\prime 2}}+3 \frac{\bar{V}_{, \sigma}^{2}}{\bar{\sigma}^{\prime 4}}+3 H \frac{\bar{V}_{, \sigma}}{\bar{\sigma}^{\prime 3}}-2 \frac{\bar{V}_{, s s}}{\bar{\sigma}^{\prime 2}}-6 \frac{\bar{\theta}^{\prime 2}}{\bar{\sigma}^{\prime 2}}\right) \delta s^{\prime 2} \delta s \\
& +\left(10 \frac{\bar{\theta}^{\prime} \bar{\theta}^{\prime \prime}}{\bar{\sigma}^{\prime 2}}+\frac{3}{2 \bar{\sigma}^{\prime}} \bar{V}_{, s s \sigma}+5 \frac{\bar{V}_{, \sigma} \bar{V}_{, s s}}{\bar{\sigma}^{\prime 3}}+7 \frac{\bar{\theta}^{\prime 2} \bar{V}_{, \sigma}}{\bar{\sigma}^{\prime 3}}+3 H \frac{\bar{V}_{, s s}}{\bar{\sigma}^{\prime 2}}-14 H \frac{\bar{\theta}^{\prime 2}}{\bar{\sigma}^{\prime 2}}\right) \delta s^{\prime} \delta s^{2} \\
& -\left(\frac{1}{6} \bar{V}_{, s s s s}-\frac{7}{3} \frac{\bar{\theta}^{\prime}}{\bar{\sigma}^{\prime}} \bar{V}_{, s s s}+2 \frac{\bar{V}_{, s s}^{2}}{\bar{\sigma}^{\prime 2}}+21 \frac{\bar{\theta}^{\prime 2} \bar{V}_{, s s}}{\bar{\sigma}^{\prime 2}}+27 \frac{\bar{\theta}^{\prime} 4}{\bar{\sigma}^{\prime 2}}\right) \delta s^{3} .
\end{aligned}
$$

[1] J. M. Bardeen, Phys. Rev. D 22, 1882 (1980).

[2] H. Kodama and M. Sasaki, Prog. Theor. Phys. Suppl. 78, 1 (1984).

[3] V. F. Mukhanov, H. A. Feldman and R. H. Brandenberger, Phys. Rept. 215, 203 (1992).

[4] R. Durrer, Fund. Cosmic Phys. 15, 209 (1994) [arXiv:astro-ph/9311041].

[5] D. Langlois, "Inflation, quantum fluctuations and cosmological perturbations", Cargese lectures 2003 [arXiv:hepth/0405053].

[6] K. A. Malik and D. Wands, Phys. Rept. 475, 1 (2009) [arXiv:0809.4944 [astro-ph]].

[7] D. Langlois, "Lectures on inflation and cosmological perturbations," arXiv:1001.5259 [astro-ph.CO].

[8] K. Tomita, Prog. Theor. Phys. 37, 831 (1967).

[9] M. Bruni, S. Matarrese, S. Mollerach and S. Sonego, Class. Quant. Grav. 14, 2585 (1997) [arXiv:gr-qc/9609040].

[10] S. Matarrese, S. Mollerach and M. Bruni, Phys. Rev. D 58, 043504 (1998) [arXiv:astro-ph/9707278].

[11] N. Bartolo, E. Komatsu, S. Matarrese and A. Riotto, Phys. Rept. 402, 103 (2004) [arXiv:astro-ph/0406398].

[12] H. Noh and J. c. Hwang, Phys. Rev. D 69, 104011 (2004).

[13] D. S. Salopek and J. R. Bond, Phys. Rev. D 42, 3936 (1990).

[14] G. L. Comer, N. Deruelle, D. Langlois and J. Parry, Phys. Rev. D 49, 2759 (1994).

[15] N. Deruelle and D. Langlois, Phys. Rev. D 52, 2007 (1995) [arXiv:gr-qc/9411040].

[16] D. H. Lyth and D. Wands, Phys. Rev. D 68, 103515 (2003) [arXiv:astro-ph/0306498].

[17] G. I. Rigopoulos and E. P. S. Shellard, Phys. Rev. D 68, 123518 (2003) [arXiv:astro-ph/0306620].

[18] E. W. Kolb, S. Matarrese, A. Notari and A. Riotto, Mod. Phys. Lett. A 20, 2705 (2005) [arXiv:astro-ph/0410541].

[19] D. H. Lyth, K. A. Malik and M. Sasaki, JCAP 0505, 004 (2005) [arXiv:astro-ph/0411220].

[20] A. A. Starobinsky, JETP Lett. 42, 152 (1985) [Pisma Zh. Eksp. Teor. Fiz. 42, 124 (1985)].

[21] M. Sasaki and E. D. Stewart, Prog. Theor. Phys. 95, 71 (1996) [arXiv:astro-ph/9507001].

[22] D. Langlois and F. Vernizzi, Phys. Rev. Lett. 95, 091303 (2005) [arXiv:astro-ph/0503416].

[23] D. Langlois and F. Vernizzi, Phys. Rev. D 72, 103501 (2005) [arXiv:astro-ph/0509078].

[24] D. Langlois and F. Vernizzi, JCAP 0602, 014 (2006) [arXiv:astro-ph/0601271].

[25] D. Langlois and F. Vernizzi, JCAP 0702, 017 (2007) [arXiv:astro-ph/0610064].

[26] S. W. Hawking, Astrophys. J. 145, 544 (1966).

[27] G. F. R. Ellis, Relativistic Cosmology, in General Relativity and Cosmology, proceedings of the XLVII Enrico Fermi Summer School, edited by R. K. Sachs (Academic, New York, 1971).

[28] G. F. R. Ellis and M. Bruni, Phys. Rev. D 40, 1804 (1989).

[29] G. F. R. Ellis, J. Hwang and M. Bruni, Phys. Rev. D 40, 1819 (1989).

[30] G. F. R. Ellis, M. Bruni and J. Hwang, Phys. Rev. D 42, 1035 (1990).

[31] M. Bruni, G. F. R. Ellis and P. K. S. Dunsby, Class. Quant. Grav. 9, 921 (1992).

[32] P. K. S. Dunsby, M. Bruni and G. F. R. Ellis, Astrophys. J. 395, 54 (1992). 
[33] M. Bruni, P. K. S. Dunsby and G. F. R. Ellis, Astrophys. J. 395, 34 (1992).

[34] R. Maartens, this issue.

[35] C. A. Clarkson, Phys. Rev. D 70, 103524 (2004) [Erratum-ibid. D 70, 129902 (2004)] [arXiv:astro-ph/0311505].

[36] J. M. Bardeen, P. J. Steinhardt and M. S. Turner, Phys. Rev. D 28, 679 (1983).

[37] J. C. Hwang, Astrophys. J. 380, 307 (1991).

[38] D. Wands, K. A. Malik, D. H. Lyth and A. R. Liddle, Phys. Rev. D 62, 043527 (2000) [arXiv:astro-ph/0003278].

[39] R. M. Wald, Chicago, Usa: Univ. Pr. (1984) 491p

[40] K. A. Malik and D. Wands, Class. Quant. Grav. 21, L65 (2004) [arXiv:astro-ph/0307055].

[41] D. H. Lyth and Y. Rodriguez, Phys. Rev. D 71, 123508 (2005) [arXiv:astro-ph/0502578].

[42] K. Enqvist, J. Hogdahl, S. Nurmi and F. Vernizzi, Phys. Rev. D 75, 023515 (2007) [arXiv:gr-qc/0611020].

[43] J. L. Lehners and S. Renaux-Petel, Trispectrum," Phys. Rev. D 80, 063503 (2009) [arXiv:0906.0530 [hep-th]].

[44] D. Langlois and S. Renaux-Petel, JCAP 0804, 017 (2008) [arXiv:0801.1085 [hep-th]].

[45] S. Renaux-Petel and G. Tasinato, JCAP 0901, 012 (2009) [arXiv:0810.2405 [hep-th]].

[46] C. Gordon, D. Wands, B. A. Bassett and R. Maartens, Phys. Rev. D 63, 023506 (2001) [arXiv:astro-ph/0009131].

[47] S. Groot Nibbelink and B. J. W. van Tent, Class. Quant. Grav. 19, 613 (2002) [arXiv:hep-ph/0107272].

[48] G. I. Rigopoulos, E. P. S. Shellard and B. J. W. van Tent, Phys. Rev. D 73, 083521 (2006) [arXiv:astro-ph/0504508].

[49] C. Pitrou and J. P. Uzan, Phys. Rev. D 75, 087302 (2007) [arXiv:gr-qc/0701121].

[50] M. Sasaki, Prog. Theor. Phys. 76, 1036 (1986).

[51] V. F. Mukhanov, Sov. Phys. JETP 67, 1297 (1988) [Zh. Eksp. Teor. Fiz. 94N7, 1 (1988)].

[52] A. Taruya and Y. Nambu, Phys. Lett. B 428, 37 (1998) [arXiv:gr-qc/9709035].

[53] V. Acquaviva, N. Bartolo, S. Matarrese and A. Riotto, Nucl. Phys. B 667, 119 (2003) [arXiv:astro-ph/0209156].

[54] F. Vernizzi, Phys. Rev. D 71 (2005) 061301 [arXiv:astro-ph/0411463].

[55] K. A. Malik, JCAP 0511, 005 (2005) [arXiv:astro-ph/0506532].

[56] K. A. Malik, JCAP 0703, 004 (2007) [arXiv:astro-ph/0610864].

[57] D. H. Lyth and Y. Rodriguez, Phys. Rev. Lett. 95, 121302 (2005) [arXiv:astro-ph/0504045].

[58] F. Vernizzi and D. Wands, JCAP 0605, 019 (2006) [arXiv:astro-ph/0603799].

[59] J. Maldacena, JHEP 0305, 013 (2003) [arXiv:astro-ph/0210603].

[60] D. Wands, this issue.

[61] D. Langlois, F. Vernizzi and D. Wands, JCAP 0812, 004 (2008) [arXiv:0809.4646 [astro-ph]].

[62] K. Koyama, arXiv:1002.0600 [hep-th].

[63] D. Langlois, S. Renaux-Petel, D. A. Steer and T. Tanaka, Phys. Rev. Lett. 101, 061301 (2008) [arXiv:0804.3139 [hep-th]]

[64] C. T. Byrnes and D. Wands, Phys. Rev. D 74, 043529 (2006) [arXiv:astro-ph/0605679].

[65] Z. Lalak, D. Langlois, S. Pokorski and K. Turzynski, JCAP 0707, 014 (2007) [arXiv:0704.0212 [hep-th]].

[66] C. T. Byrnes, M. Sasaki and D. Wands, Phys. Rev. D 74, 123519 (2006) [arXiv:astro-ph/0611075].

[67] D. Seery, M. S. Sloth and F. Vernizzi, JCAP 0903, 018 (2009) [arXiv:0811.3934 [astro-ph]].

[68] C. T. Byrnes, K. Y. Choi and L. M. H. Hall, JCAP 0810, 008 (2008) [arXiv:0807.1101 [astro-ph]].

[69] D. Seery and J. E. Lidsey, JCAP 0701, 008 (2007) [arXiv:astro-ph/0611034].

[70] C. T. Byrnes and K. Y. Choi, arXiv:1002.3110 [astro-ph.CO].

[71] F. Bernardeau and J. P. Uzan, Phys. Rev. D 66, 103506 (2002) [arXiv:hep-ph/0207295].

[72] G. I. Rigopoulos, E. P. S. Shellard and B. J. W. van Tent, Phys. Rev. D 73, 083522 (2006) [arXiv:astro-ph/0506704].

[73] F. Bernardeau, this issue.

[74] J. L. Lehners and P. J. Steinhardt, Phys. Rev. D 77, 063533 (2008) [Erratum-ibid. D 79, 129903 (2009)] [arXiv:0712.3779 [hep-th]].

[75] J. L. Lehners and P. J. Steinhardt, Phys. Rev. D 78, 023506 (2008) [Erratum-ibid. D 79, 129902 (2009)] [arXiv:0804.1293 [hep-th]].

[76] J. L. Lehners, arXiv:1001.3125 [hep-th].

[77] K. Koyama, S. Mizuno, F. Vernizzi and D. Wands, JCAP 0711, 024 (2007) [arXiv:0708.4321 [hep-th]].

[78] K. Enqvist and A. Vaihkonen, "Non-Gaussian perturbations in hybrid inflation," JCAP 0409, 006 (2004) [arXiv:hep$\mathrm{ph} / 0405103]$.

[79] A. Jokinen and A. Mazumdar, JCAP 0604, 003 (2006) [arXiv:astro-ph/0512368].

[80] N. Barnaby and J. M. Cline, Phys. Rev. D 73, 106012 (2006) [arXiv:astro-ph/0601481].

[81] N. Barnaby and J. M. Cline, Phys. Rev. D 75, 086004 (2007) [arXiv:astro-ph/0611750].

[82] D. Langlois, Phys. Rev. D 59, 123512 (1999) [arXiv:astro-ph/9906080].

[83] E. Komatsu et al., arXiv:1001.4538 [astro-ph.CO].

[84] M. Kawasaki, K. Nakayama, T. Sekiguchi, T. Suyama and F. Takahashi, JCAP 0811, 019 (2008) [arXiv:0808.0009 [astro$\mathrm{ph}]$.

[85] M. Kawasaki, K. Nakayama, T. Sekiguchi, T. Suyama and F. Takahashi, JCAP 0901, 042 (2009) [arXiv:0810.0208 [astro$\mathrm{ph}]$.

[86] E. Kawakami, M. Kawasaki, K. Nakayama and F. Takahashi, JCAP 0909, 002 (2009) [arXiv:0905.1552 [astro-ph.CO]].

[87] T. Takahashi, M. Yamaguchi and S. Yokoyama, Phys. Rev. D 80, 063524 (2009) [arXiv:0907.3052 [astro-ph.CO]].

[88] D. Langlois and F. Vernizzi, Phys. Rev. D 70, 063522 (2004) [arXiv:astro-ph/0403258]. 